\author{
M. Month
}

March 26, 1984

\begin{abstract}
DISCLAIMER
This report was prepared as an account of work sponsored by an agency of the United States Government. Neither the United States Government nor any agency thereof, nor any of their employees, makes any warraniy, express or implied, or assumes any legal liability or responsjbility for the accuracy, completeness, or usefulness of any information, apparatus, product, or process disclosed, or represents that its use would not infringe privately owned rights. Reference herein to any specific commercial product, process, or service by trade name, trademark, manufacturer, or otherwise does not necessarily constitute or imply its endorsement, recom. mesdation, or favoring by the United States Government or any agency thereof. The views and opinions of authors expressed herein do not necessarily state or reflect those of the United States Government or any agency thereof,
\end{abstract}

ACCELERATOR DEPARTMENT

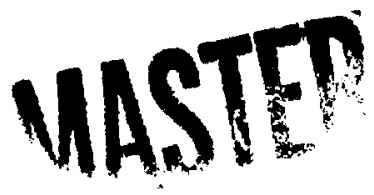

BROOKHAVEN NATIONAL LABORATORY

UPTON, LONG ISLAND, NEW YORK 11973 
i.

This report was prepared as an accourit of work sponsored by an agency of the United States Government. Neither the United Stntes Government nor any agency thereof, nor any of their employees, nor any of their contractors, subcontractors, or their employees, makes any warranty, express or implier, or assumes any legal liability or responsibility for the accuracy, completeness, or usefulness of any information, apparatus, product, or process disclosed, or represents that its use would not infringe privately owned rights. Reference herein to any specific commercial product, process, or service by trade name, trademark, manufacturer, or otherwise, does not necessarily constitute or imply its endorsement, recommendation, or favoring by the United States Government or any agency, contractor or subcontractor thereof. The views and .opinions of authors expressed herein do not necessarily state or reflect those of the United States Government or any agency, contractor or subcontractor thereof.

Printed in the United States of America

Available from

National Technical Information Service

U.S. Department of Commerce

5285 Port Royal Road

Springfield, VA 22161

NTIS price codes:

Printed Copy: A02; Microfiche Copy: A01 


\title{
Beam Model for Non-Planar Orbits in Synchrotrons
}

\author{
M. Month
}

March 26, 1984

\begin{abstract}
A framework has been developed for a beam model in the case of synchrotron orbits not confined to a plane. An appropriate moving reference system for the analysis of beam stability has been introduced. As examples of strong perturbations to median plane symmetry, two geometries for the overpass for the Tevatron collider are considered.
\end{abstract}




\author{
Beam Model For \\ Non-P lanar Orbits In Synchrotxons \\ M. Month \\ March 26, 1984
}

\title{
1. Introduction
}

A moving reference frame appropriate for the analysis of beam stability in E strong focusing synchrotron for a planar reference orbit has been extensively used. (1) Here, we introduce a moving reference system for a non-planar orbit, analyzing the case of a non-planar perturbation to a given planar orbit. Thus, we develop a framework for a beam model to describe particle motion for nonplanar orbits, as in the overpass configuration.

The formalism for the Beam Model in space includes:

1. The Introduction of a General Periodic Moving Reference Frame in space;

2. A procedure for following in space the central reference orbit and the local reference frame;

3. An analysis of tr methods of magnet placement on the up and down slopes - the Great Circle Method and The Screw Method;

4. An analysis of quadrupole placement in the perturbed region;

5. A procedure for setting up the equations for particle oscillations about the central orbit; and

6. A qualitative description of the nature of the coupling introduced when magnet orientation is constrained.

1. See, for example, E.D. Courant and H.S. Snyder, Annals of Physics 3, 1 (1958).

2. See, S. Ohnuma, Fermilab Report TM-1124 (1982) and T. Collins, unpublished (1981). 


\section{Transformations Through Elements}

There are two types of transformations to consider.

Particle motion in a beam can be described in terms of a local Cartesian coordinate frame of reference. This moving frame of reference can be defined uniquely by the condition that the reference particle (orbit) have all its bending in dipoles removed from the equations of motion. Another way of phrasing this condition is that the set of unit vectors defining the local frame transforms continuously through dipoles in the manner of a set of velocity vectors, that is, a rotation about the direction of the magnetic field by an angle equal to the bending angle of the magnet. In this reference system, the transformation of the local coordinate frame through a quadrupole or drift is the unit transformation.

A second transformation relates to the positon of the reference orbit (particle) in space. In a dipole the effective change in position of the central particle is along the chord defining the orbit through the magnet and a distance equal to the chord length. If the magnet is oriented so that the chord represents the magnet axis (perpendicular to the field), then the change of position is the magnet length along the magnet axis. In a quadrupole or drift, the change in position is just the length of the element along the direction of the central orbit (assuming the quadrupole axis is located along the orbit).

As the coordinate frame is transformed through the elements defining the accelerator, we follow the vector defining the motion of the central particle. Transverse to this direction, the central orbit has no motion. However, a beam of particles is a set of particles oscillating transversely about the central orbit. Each particle is therefore defined in terms of its transverse velocities. We therefore refer to the transverse coordinates. To keep track of 
the transformation of the transverse coordinates, we maintain the expression "vertical" to refer to the transverse direction which corresponds to the actual vertical when the orbit motion is in the accelerator median plane. Since the coordinate transformation is continuous, we can define "vertical" uniquely, even though the actual direction of this transverse coordinate is deflected from the direction perpendicular to the accelerator median plane.

Even in the case when magnets (dipoles and quadrupoles) are placed with their magnetic fields perpendicular to the direction of the central orbit, we must specify the angle which defines the direction in the transverse plane. For a dipole, defin the angle between the field direction and the "vertical" of the reference system to accomplish this. In a quadrupole, the relevant angle is the angle between the "vertical" and the field direction corresponding to vertical focusing. In this latter instance, if the two "vertical" directions are not the same, the quadrupole will introduce vertical-horizontal coupling into the transverse motion of particles in the beam.

The Local Coordinate System and General Transformations

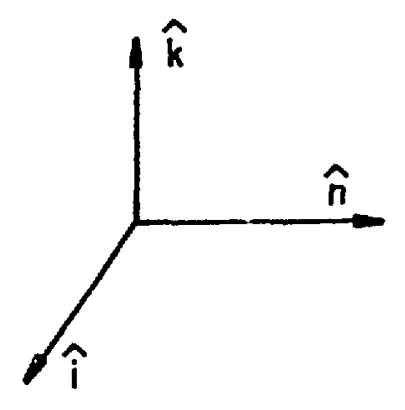

Figure 1: Right-handed Coordinate Frame of Reference. Unit Vector Set $(\hat{k}, \hat{i}, \hat{n})$.

Let $(\hat{k}, \hat{i}, \hat{n})$ represent a set of unit vectors defining a righthanded coordinate frame of reference. The "hat" is used for unit vectors. 
Let us refer to this set of unit vectors or to any one of the vectors comprising the set by $\hat{v}$. Then a transformation is written,

$$
(\hat{k}, \hat{i}, \hat{n}) \rightarrow\left(\hat{k}^{\prime}, \hat{i}^{\prime}, \hat{n}^{\prime}\right)
$$

or

$$
\hat{\mathbf{v}}+\hat{\mathbf{v}}^{\prime}
$$

The transformation through an element is in general a rotation. However, through a quadrupole (or drift) the transformation is a zero angle rotation or a unit transformation. Referring to this transformation as the unit transformation, I, we have through a drift or quadrupole,

$$
\hat{v}^{\prime}=I \hat{V}
$$

To keep track of quadrupoles, we use the unit transformation symbol $I_{\hat{q}}$ when a quadrupole is involved, where $\hat{q}$ is the diraction of quadrupole vertical focusing. Through a dipole, the transformation of the reference vectors is a rotation about the magnetic field direction, $\hat{b}$, of magnitude equal to the bending angle, $\Phi$. Referring to this transformation by the symbol $\mathrm{R}_{\mathrm{b}} \Phi$, we have

$$
\hat{\mathbf{v}}^{\prime}=\mathrm{R}_{\hat{\mathrm{b}}, \Phi} \hat{\mathbf{v}}
$$

This representation can be made operational for the set of coordinate system unit vectors, if $\hat{v}$ is interpreted as a 3 -component vector comprised of the three coordinate axis unit vectors and $R$ and $I$ are $3 \times 3$ matrices. Thus, we can write general transformation through $M$ components as

$$
\hat{v}^{\prime}=R_{M} R_{M-1} \cdots \cdots \cdot R_{2} R_{1} \hat{v}
$$


In the case of a circular accelerator of $M$ elements, we must impose the periodicity condition for the reference frame:

$$
\hat{v}^{\prime}=\hat{v}=R_{M} R_{M-1} \cdots \cdot R_{2} R_{1} \hat{v}
$$

\section{Transformations Through a Dipole Magnet}

The transformation of the reference system through a dipole is described in terms of the magnetic field direction, $\hat{b}$, and the bending angle (in the dipole plane), $\Phi$. Let $\bar{B}$ be the magnetic field strength. Then, if $B p$ is the magnetic rigidity (momentum of the reference particle) and $\ell_{B}$ is the path length through the magnet in the magnet plane, then the bending angle is given by,

$$
\Phi=\frac{\overline{\mathrm{B} \ell_{B}}}{\mathrm{~B} \rho},
$$

where $B$ and $\rho$ are usually chosen to refer to the magnets which are used as the repetitive building blocks to form the accelerator. Thus, the radius of curvature in the magnet is

$$
\bar{\rho}=\frac{B \rho}{\bar{B}},
$$

and the chord length in the plane of the magnet is

$$
2 \mathrm{~d}=2 \bar{\rho} \sin \Phi / 2
$$

In the case where the magnetic field is perpendicular to the incoming orbit direction, then the change in the position vector (t) is given by,

$$
\Delta \vec{t}=(2 d) \hat{m}
$$


where $\hat{m}$ is the unit vector along the magnet. symmetry axis direction and we are free to assume that the magnet is oriented so that the incoming orbit direction is at an angle $\Phi / 2$ to the symmetry axis in the magnet plane. In this configuration, (2d) is the length of the dipole, $\ell_{D}$, and $\Phi$ is given by

$$
\Phi=2 \sin ^{-1}\left(\frac{l \mathrm{D}}{2 \rho}\right)
$$

Also the change in $\$$ is

$$
\Delta t=l_{\mathrm{D}} \hat{\mathbf{m}}
$$

The reference system $\hat{v}$ transforms through a dipole by the rotation $R_{\hat{b}, \Phi}$.

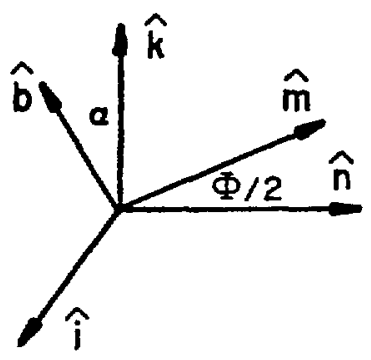

Figure 2: Angle (a) Between Local Beam Frame and Magnetic Field

The vector $\hat{b} \perp \hat{n}$ and $\alpha$ is the angle with respect to the vertical:

$$
\hat{b} \cdot \hat{k}=\cos \alpha \text {. }
$$

From the general expression for a vector rotation about $\hat{b}$ by an angle $\Phi$, given by ,

$$
\hat{v}^{\prime}=\cos \Phi \hat{v}+\sin \Phi(\hat{b} \times \hat{v})+(1-\cos \Phi)(\hat{b} \cdot \hat{v}) \hat{b}
$$

we can show that 


$$
\left(\begin{array}{c}
\hat{n}^{\prime} \\
\hat{k}^{\prime} \\
\hat{i}^{\prime}
\end{array}\right)=R_{\hat{b}, \Phi}\left(\begin{array}{l}
\hat{\mathrm{r}} \\
\hat{k} \\
i
\end{array}\right)
$$

with,

$$
R_{\hat{b}, \Phi}=\left(\begin{array}{lll}
\cos \Phi & \sin \phi \sin \alpha & -\sin \phi \cos \alpha \\
-\sin \phi \sin \alpha & 1-(1-\cos \phi) \sin ^{2} \alpha & (1-\cos \phi) \sin \alpha \cos \alpha \\
\sin \phi \cos \alpha & (1-\cos \phi) \sin \alpha \cos \alpha & 1-(1-\cos \Phi) \cos ^{2} \alpha
\end{array}\right) .
$$

The magnet orientation is chosen such that its symmetry axis is at an angle $\Phi / 2$ to the incoming vector, $\hat{n}$, so that

$$
\hat{\mathrm{m}}=\cos \Phi / 2 \hat{\mathrm{n}}+\sin \Phi / 2(\hat{\mathrm{b}} \times \hat{\mathrm{n}}) \text {, }
$$

constructed from

$$
\hat{\mathrm{n}} \bullet \hat{\mathrm{m}}=\cos \Phi / 2,
$$

and

$$
\hat{b}=\frac{\hat{n} x \hat{m}}{\sin \Phi / 2} \cdot
$$

Thus, we have

$$
\hat{m}=\cos \Phi / 2 \hat{n}+\sin \Phi / 2 \sin \alpha \hat{k}-\sin \Phi / 2 \cos \alpha \hat{i} .
$$

The angle $\alpha$, defined by $\hat{b} \cdot \hat{k}=\cos \alpha$, is a function not only of the direction of $\hat{b}$ in space but is a cumulative effect of the orientation of $\hat{k}$ due to the sequence of previous transformations.

The set of vectors $\hat{b}$ are in general chosen to fit a variety of geometrical constraints. Sometimes there is a sequence of dipoles which fits a 
prescribed rule of configuration. Two such sets have been termed the "Great Circle" sequence and the "Screw" sequence. In the former case, the magnetic field direction is maintained constant for the magnets in the sequence,

$$
\hat{b}=\hat{b}_{0} \text {, constant. }
$$

For the "screw" sequence, the vector $\hat{b}$ changes from magnet to magnet in the sequence by a rotation about some given direction. Let $\hat{z}$ be this direction, chosen to correspond to the "vertical" direction of the accelerator median plane. Thus, we have two planes, the magnet plane, defined by $\hat{b}$, and the accelerator median plane, defined by $\hat{z}$. Let $\delta$ be the angle between these two planes:

$$
\hat{b} \cdot \hat{z}=\cos \delta \text {. }
$$

Now, the "screw" sequence is defined so that $\hat{b}$ rotates about $\hat{z}$ from magnet to magnet by an angle $\Delta$,

$$
\hat{\mathbf{b}}^{\prime}=\mathbf{R}_{\mathbf{z}, \Delta} \hat{\mathbf{b}}
$$

The angle $\Delta$ is determined from the amount of rotation of the orbit through the magnet. If we rotate the magnet symmetry axis around $\hat{z}$ by the same amount $\Delta$, and determine $\Delta$ from the rotation of the central particle direction about $\hat{z}$, then the configuration of central orbit, magnet symmetry axis and magnetic field direction will be identical to that of the previous magnet. This is the "screw" sequence. Let us now find $\Delta$ from the rotation of $\hat{n}$ about $\hat{b}$ by the magnet bending angle, $\Phi$. 


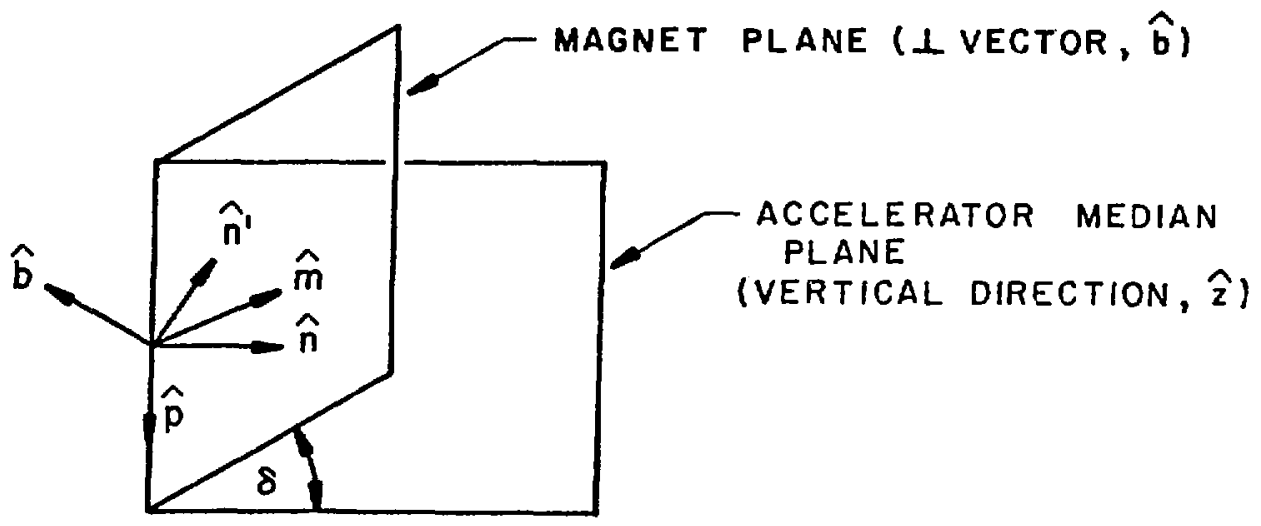

Figure 3: Accelerątgr Frame (Median Plane) and Magnet Plàne with Unit Vector Set $(b, m, p)$

In the above figure, we have that

$\hat{n}$ is the direction of the orbit "in" and

$\hat{\mathrm{n}}^{\prime}$ is the direction of the orbit "out". The vector set

$(\hat{b}, \hat{m}, \hat{p})$ represents a right-handed frame for the magnet.

With $\delta$ the angle between the 2 planes,

$$
\hat{z} \cdot \hat{b}=\cos \delta, \hat{z} \cdot \hat{m}=\sin \delta
$$

Let $\theta_{V}$ be the angle of $\hat{n}$ and $\hat{n}^{\prime}$ projected onto the accelerator median plane. (They have the same such angle by symetry.) So,

$$
\hat{z} \cdot \hat{n}=\hat{z} \cdot \hat{n}^{\prime}=\sin \theta_{V}
$$

Now, since $\hat{\mathrm{n}}=\cos \phi / 2 \hat{\mathrm{m}}+\sin \phi / 2 \hat{\mathrm{p}}$, we have, taking the scalar product with $\hat{z}$,

$$
\sin \theta_{V}=\cos \Phi / 2 \sin \delta .
$$


Also, writing

$$
\hat{\mathbf{n}}=\sin \theta_{\mathrm{V}} \hat{z}+\cos \theta_{\mathrm{V}} \hat{\mathrm{n}}^{*} \text {, }
$$

and

$$
\hat{\mathrm{n}}^{\prime}=\sin _{\mathrm{V}} \hat{\mathrm{z}}+\cos \theta_{\mathrm{V}} \hat{\mathrm{n}}^{\prime *},
$$

where $\hat{n}^{*}$ and $\hat{n}^{\prime *}$ are the unit vector projections of $\hat{n}$ and $\hat{n}^{\prime}$ in the accelerator median plane, and taking the scalar product $\hat{n}^{\bullet} \hat{n}^{\prime}$, we can show that

$$
\sin \Delta / 2=\sin \Phi / 2 / \cos \theta_{V}
$$

Thus, in the screw sequence, $\hat{b}$ changes from magnet to magnet in the manner,

$$
\hat{b}^{\prime}=\cos \Delta \hat{b}+\sin \Delta(\hat{z} \times \hat{b})+(1-\cos \Delta)(\hat{z} \cdot \hat{b}) \hat{z},
$$

where $\Delta$ is given by,

$$
\sin \Delta / 2=\sin \Phi / 2 / \cos \theta_{V}
$$

and $\theta_{v}$ and $\delta$ are related by

$$
\sin \theta_{v}=\cos \Phi / 2 \sin \delta
$$

The symmetry axis $\hat{m}$ for the next magnet in the sequence is found in the same way as a rotation about $\hat{z}$ by the angle $\Delta$.

Transformations Through A Quadrupole Magnet

The transformation of the reference system through a quadrupole is the same as through a drift, the unit transformation. The change in the position vector $t$ is also the same as through a drift element. If $\ell_{Q}$ is the quadrupole length and $\hat{n}$ is the orbit direction unit vector, then

$$
\Delta \neq=\ell_{Q} \hat{n}
$$


A quadrupole magnet is also defined by its orientation about the central orbit direction. Let $\hat{q}$ be a unit vector along the vertical quadrupole axis. Then the angle $Y$, which is the angle between the vertical reference vector and the vertical quadrupole axis, is given by

$$
\hat{q} \cdot \hat{k}=\cos \gamma
$$

If $\gamma \neq 0$, i.e., $\hat{q} \neq \hat{k}$, then this quadrupole orientation introduces linear coupling into the transverse motion.

III. Method of Computation

We consider the case of a median plane accelerator perturbed in a specified region to produce a space orbit not limited to the original accelerator plane. We take the reference frame in the unperturbed region to be the usual loral frame moving along the median plane orbit, with

$\hat{z}$ a unit vector perpendicular to the plane of the motion, $\hat{y}$ a unit vector in the direction of the motion, and $\hat{x}$ a unit vector perpendicular to the motion and in the plane.

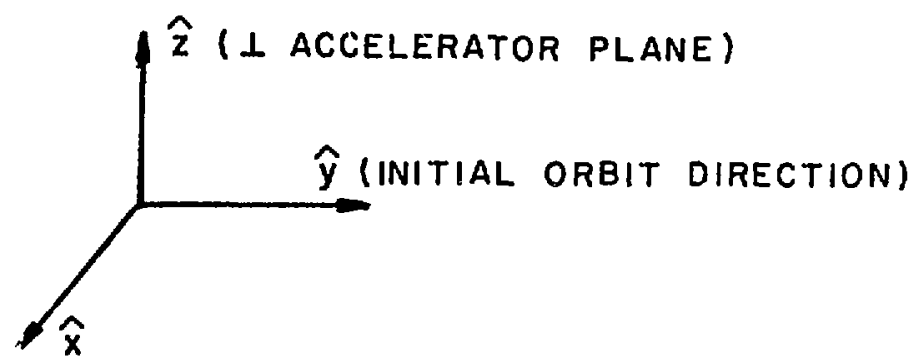

Figure 4: Loca1 Beam Frame In Unperturbed Region

For the space orbit, we take the local coordinate frame, derived continuously from the initial frame, to be the unique frame transforming the reference vectors through all pure bending elements (dipole magnets) like velocity 
vectors. In this way, the bending of the reference (centra1) orbit is incorporated in the moving coordinate system and these bending fields for the central orbit particle do not appear in the equations of motion. The continuous transformations of the local reference vectors, designated $(\hat{k}, \hat{i}, \hat{n})$, are

$$
(\hat{z}, \hat{x}, \hat{y}) \rightarrow(\hat{k}, \hat{i}, \hat{n})
$$

The unit vector $\hat{\mathbf{n}}$ is the direction of the reference orbit in space and $\hat{\mathbf{k}}$ represents the generalized "vertical" direction corresponding to $\hat{z}$ in the unperturbed regions of the accelerator.

The sequence of transformations in the perturbed region are:

o Initial Frame of Reference

I Bend up

II Up the slope

III Bend down - so that orbit is parallel to accelerator plane

IV Motion in the Straight Section - in a line parallel to the accelerator plane

$\checkmark$ Bend down

VI Down the slope

VII Bend up - Recover Initial Frame

The transformations in regions $I, I I I, V$ and VII are accomplished by choosing $\hat{b}$ to be almost perpendicular to $\hat{z}, i . e \cdot \alpha=90^{\circ}$. However, it is convenient to put a small bend to move the orbit inward to compensate the increased central orbit length caused by moving up and down. Thus, we take for these regions the following values for the magnetic field:

$$
\begin{aligned}
& \text { I } B_{\mathrm{ul}} \text { and } \alpha=90^{\circ}-\tau_{\mathrm{ul}} \\
& \text { III } \mathrm{B}_{\mathrm{dl}} \text { and } \alpha=-90^{\circ}+\tau_{\mathrm{dl}}
\end{aligned}
$$




$$
\begin{aligned}
& \text { v } B_{d 2} \text { and } \alpha=-90^{\circ}+\tau_{\mathrm{d} 2} \\
& \text { vII } B_{\mathrm{u} 2} \text { and } \alpha=90^{\circ}-\tau_{\mathrm{u} 2}{ }^{\circ}
\end{aligned}
$$

The $B$ 's and $\tau^{\prime} s$ are chosen

to provide the required slope angle,

to provide the required separation in the straight section,

to provide the final orbit and reference frame match to the unperturbed

region, and

to provide an unchanged reference orbit length.

In regions II and VI, we are woving up and down the slope, respectively.

In these regions, the magnetic field strengths are taken to be the normal

values, i.e. in the absence of the overpass perturbation. Thus, we must

choose only $\alpha$ for each magnet. We consider two magnet sequences on the slopes:

The Great Circle sequence and the Screw sequence.

The Great Circle Sequence

Choose $\hat{b}=\hat{k}$ in each magnet on the slope. However, this choice means that $\hat{k}$ is unchanged in the whole sequence. Therefore $\hat{b}$ is unchanged in the se- ; quence. Note that $\hat{b}$ in the up slope is different from $\hat{b}$ in the down slope and the Up Great Circle and the Down Great Circle are in general different.

To obtain a matching of the perturbed region to the unperturbed, the final trans formation must accomplish

$$
(\hat{\mathrm{k}}, \hat{\mathrm{i}}, \hat{\mathrm{n}})+(\hat{\mathrm{z}}, \hat{\mathrm{x}}, \hat{\mathrm{y}}) \text { of appropriate directions. }
$$

we can see that the matching of $\hat{n}$ actually ensures the matching of $\hat{k}$. First we note that the transformation of every vector is a set of rotations and this set of rotations, by linearity, is independent of the initial vector being 
transformed. So, take the vector $\hat{y}$ just before the perturbation. Let $R_{p}$ be the matrix through the perturbation. So,

$$
\hat{y}^{\prime}=R_{p} \hat{y}
$$

For a match across the perturbed region, $\hat{y}^{\prime}$ must be pointing in the proper direction in space. We have also that $\hat{z}+\hat{z}^{\prime}$, where

$$
\hat{z}^{\prime}=R_{p} \hat{z}
$$

Let $R_{u}$ represent the rotation around the uperturbed region. Then periodicity, i.e. matching the orbit, implies that the combination

$$
R_{u} R_{p}=I \text {, the unit matrix. }
$$

However, we $k=$ what $R_{u}$ is a rotation about $\hat{z}$, since all magnets in the unperturbed region have their fields in the direction $\hat{z}$. Thus $R_{u}$ (or $R_{u}^{-1}$ ) acting on $\hat{z}$ leaves $\hat{z}$ unchanged; we therefore have,

$$
R_{u} \hat{z}^{\prime}=R_{u} R_{p} \hat{z}=\hat{z}
$$

and so,

$$
\hat{z}^{\prime}=R_{u}^{-1 \hat{z}}=\hat{z}
$$

Thus, $\hat{z}$ is matched across the perturbation. Also, $\hat{y}$ is matched by construction. Thus, $\hat{x}$ is also matched, with

$$
\hat{x}^{\prime}=R_{\mathbf{p}} \hat{x} \text {, across the perturbation. }
$$




\section{The Screw Sequence}

In the Great Circle sequence, the first element of the sequence is simply determined by setting $\hat{b}=\hat{k}$ at the input. All the other magnet placements follow by choosing, $\hat{b}=\hat{k}$ for all elements. In the screw sequence, $\hat{b}$ is not in the direction of $\hat{k}$ but is rotated from $\hat{k}$ about $\hat{n}$ by the angle $\alpha$. To fix $\alpha$, we impose the condition that the angle of projection of $\hat{n}$ onto the unperturbed accelerator plane be unchanged. Let $\theta_{\mathrm{V}}$ be this angle at the input to the screw sequence; that is, at the input,

$$
\hat{\mathrm{n}} \cdot \hat{\mathrm{z}}=\sin \theta_{\mathrm{V}}
$$

After passing through the element, $\hat{n}+\hat{n}^{\prime}$. Thus, to determine $\alpha$, we insist that $\theta_{\mathrm{V}}$ is unchanged; that is,

$$
\hat{n}^{\prime} \cdot \hat{z}=\hat{n} \cdot \hat{z}=\sin \theta_{V} \text {. }
$$

This sequence has the interesting property that the magnet plane for each magnet in the sequence and the unperturbed accelerator plane intersect at tha same angle. We have taken this angle to be $\delta$, so that

$$
\hat{b} \cdot \hat{z}=\cos \delta \text {, for a } 11 \text { magnets in the sequence. }
$$

As we have shown previously, setting the magnet so that its symetry axis is at angle $\phi / 2$ to the incoming $\hat{n}, \theta_{V}$ and $\delta$ are related by

$$
\sin \theta_{\mathrm{v}}=\sin \delta \cos \phi / 2
$$


where $\phi$ is the magnet bend angle. Furthermore, we have seen that the screw sequence is such that the field vector $\hat{b}$ rotates about $\hat{z}$ from magnet to magnet by the angle $\Delta$. That is from element $\mathrm{J}$ to element $\mathrm{J}+1$ in the sequence:

$$
\hat{\mathrm{b}}_{\mathrm{J}+1}=\cos \Delta \hat{\mathrm{b}}_{\mathrm{J}}+\sin \Delta\left(\hat{z}_{\mathrm{z}} \hat{\mathrm{b}}_{\mathrm{J}}\right)+(1-\cos \Delta)\left(\hat{z} \cdot \hat{\mathrm{b}}_{\mathrm{J}}\right) \hat{\mathrm{z}}_{\mathrm{g}}
$$

with $\Delta$ given by

$$
\sin \Delta / 2=\sin \phi / 2 / \cos \theta_{v}
$$

Thus the orientation of each magnet, in particular the vectors $\hat{b}$ and $\hat{m}$ (magnet symetry axis direction), is determined by a rotation of magnitude $\Delta$ about the direction $\hat{z}$ relative to the previous magnet in the sequence. The angle $\alpha$, the angle between the field vector $\hat{b}$ and the local vertical vector, $\hat{k}$, at the element input, is found from

$$
\hat{n} \cdot \hat{z}=\sin \theta_{y}=\hat{n} \cdot \hat{z} \text {. }
$$

Again, to match the perturbed to the unperturbed regions, the final transformation of the perturbed region must accomplish

$$
(\hat{k}, \hat{i}, \hat{n})+(\hat{z}, \hat{x}, \hat{y}) \text { of appropriate direction. }
$$

and again the reference frame matching across the perturbation is ensured by the matching of the orbit direction.

Transformation Algorithm for the Angle $\alpha$ in the Screw Sequence

At the $\mathrm{J}^{\text {th }}$ point in the sequence, let us take $\alpha_{\mathrm{J}}$ to be the angle between $\hat{\mathrm{k}}_{\mathrm{J}}$ and $\hat{\mathrm{b}}_{\mathrm{J}}$. At the input of the $(\mathrm{J}+1)^{\text {th }}$ magnet, we write $\alpha_{\mathrm{J}+1}, \hat{\mathrm{k}}_{\mathrm{J}+1}$ and $\hat{b}_{J+1}$. The rotation angle of the $\hat{b}_{J}$ vector is $\Delta$ (independent of $J$ for the sequence); that is, 


$$
\hat{\mathrm{b}}_{\mathrm{J}+1}=\cos \Delta \hat{\mathrm{b}}_{\mathrm{J}}+\sin \Delta\left(\hat{\mathrm{z} \times \hat{b}_{\mathrm{J}}}\right)+(1-\cos \Delta)\left(\hat{\mathrm{z}} \cdot \hat{\mathrm{b}}_{\mathrm{J}}\right) \hat{\mathrm{z}}
$$

where $\Delta$ is given by

$$
\sin \Delta / 2=\sin \phi / 2 / \cos \theta_{v}
$$

with $\phi$ the bend angle of each magnet in the sequence and $\theta_{v}$ related to $\delta$, the angle of each magnet plane in the sequence with the accelerator median plane, by,

$$
\sin \theta_{v}=\cos \phi / 2 \sin \delta
$$

The "vertical" vector transformation is

$$
\hat{\mathrm{k}}_{\mathrm{J}+1}=\cos \phi \hat{\mathrm{k}}_{\mathrm{J}}-\sin \phi \sin \alpha_{\mathrm{J}} \hat{\mathrm{n}}_{\mathrm{J}}+(1-\cos \phi) \cos \alpha_{\mathrm{J}} \hat{\mathrm{b}}_{\mathrm{J}}
$$

where,

$$
\hat{b}_{J} \times \hat{k}_{J}=-\sin \alpha_{J} \hat{n}_{J}
$$
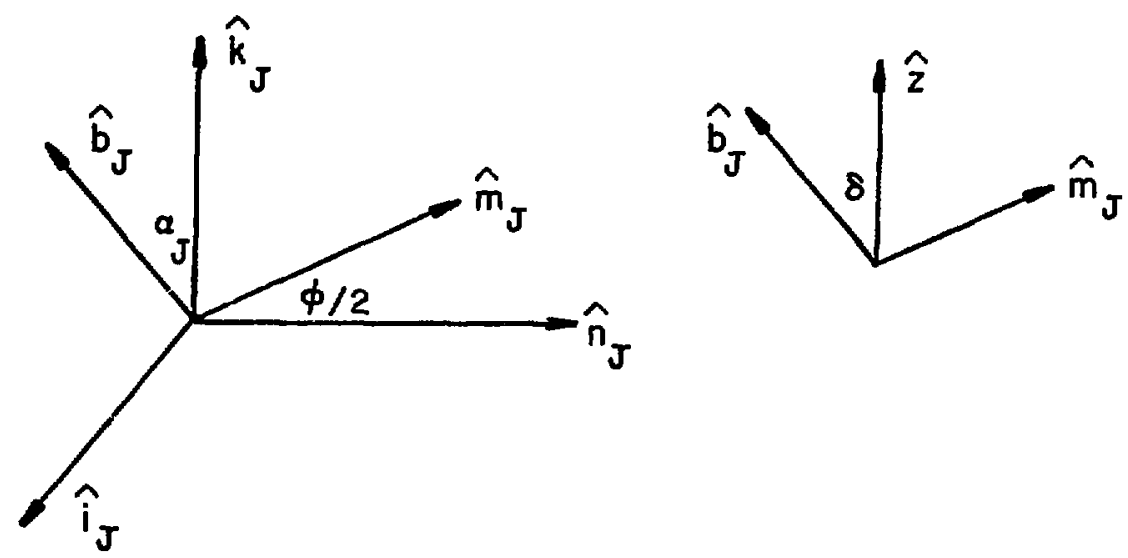

Figure 5: Angles $\left(\alpha_{J}, \phi, \delta\right)$ to Determine Local Beam Frame Orientation in Space 
We need $\hat{z}$ in terms of the local vectors. From,

$$
\hat{z}=\cos \delta \hat{b}_{J}+\sin \delta \hat{m}_{J} \text {, }
$$

and

$$
\hat{m}_{J}=\cos \phi / 2 \hat{n}_{J}+\sin \phi / 2\left(\hat{b}_{J} \times \hat{n}_{J}\right)
$$

we have,

$$
\hat{z}=\cos \delta \hat{b}_{J}+\sin \delta \cos \phi / 2 \hat{n}_{J} \cdot \sin \delta \sin \phi / 2\left(\hat{b}_{J} \hat{n}_{J}\right) .
$$

There fore,

$$
\hat{z}_{x} \hat{b}_{J}=-\sin \delta \cos \phi / 2 \hat{\mathrm{b}}_{\mathrm{J}} \times \hat{n}_{\mathrm{J}}+\sin \delta \sin \phi / 2 \hat{\mathrm{n}}_{\mathrm{J}} \text {, }
$$

where

$$
\left(\hat{\mathrm{b}}_{\mathrm{J}} \times \hat{\mathrm{n}}_{\mathrm{J}}\right) \times \hat{\mathrm{b}}_{\mathrm{J}}=\hat{\mathrm{n}}_{\mathrm{J}} \text {, }
$$

and we have

$$
\begin{aligned}
\hat{\mathrm{b}}_{\mathrm{J}+1}= & \left(1-\sin ^{2} \delta(1-\cos \Delta)\right) \hat{\mathrm{b}}_{\mathrm{J}} \\
& +(\sin \Delta \sin \delta \sin \phi / 2+(1-\operatorname{ccs} \Delta) \cos \delta \sin \delta \cos \phi / 2) \hat{\mathrm{n}}_{\mathrm{J}}
\end{aligned}
$$

$+(-\sin \Delta \sin \delta \cos \phi / 2+(1-\cos \Delta) \cos \delta \sin \delta \sin \phi / 2) \hat{\mathrm{b}}_{\mathrm{J}} \times \hat{\mathrm{n}}_{\mathrm{J}}$,

with $\hat{z} \cdot \hat{\mathrm{b}}_{\mathrm{J}}=\cos \delta$.

Using $\hat{\mathrm{b}}_{\mathrm{J}} \cdot \hat{\mathrm{k}}_{\mathrm{J}}=\cos \alpha_{\mathrm{J}}$ and $\left(\hat{\mathrm{b}}_{\mathrm{J}} \times \hat{n}_{\mathrm{J}}\right) \cdot \hat{\mathrm{k}}_{\mathrm{J}}=\sin \alpha_{\mathrm{J}}$, we obiain

$$
\begin{gathered}
\hat{\mathrm{b}}_{\mathrm{J}+1} \cdot \hat{\mathrm{k}}_{\mathrm{J}+1}=\cos \alpha_{\mathrm{J}+1}=\cos \alpha_{\mathrm{J}}\left(1-(1-\cos \Delta) \sin ^{2} \delta\right) \\
-\sin \alpha_{\mathrm{J}} \sin \Delta \sin \delta \cos \phi / 2-\sin \alpha_{\mathrm{J}}(1-\cos \Delta) \sin \delta \cos \delta \sin \phi / 2 .
\end{gathered}
$$

In the case of small angles $\Delta, \delta$ and $\phi$, we can expand the angles and find the lowest order algorithm. We find,

$$
\cos \alpha_{\mathrm{J}+1}=\cos \alpha_{\mathrm{J}}-\sin \alpha_{\mathrm{J}}(\Delta \delta)
$$


which is 2 nd order in the small angles. To this order, the algorithm can be written in the form,

$$
\alpha_{\mathrm{J}+1}=\alpha_{\mathrm{J}}+\Delta \delta
$$

which can be solved, giving

$$
\alpha_{\mathrm{J}}=\alpha_{0}+\mathrm{J} \Delta \delta
$$

Thus, if $\delta$ is roughly the inverse of the number of elements in the sequence, $\alpha_{\max } \approx \alpha_{0}+\Delta$, i.e. first order in a small angle.

Note that going down the slope, $\delta$ changes sign and $\alpha$ decreases roughly from $\alpha_{\max }$ back to $\alpha_{0}$.

Quadrupole Placement

The quadrupole orientation is determined by the unit vector $\hat{q}$, defining the intrinsic quadrupole vertical axis. A quadrupole is always placed with its symmetry axis in the direction of the central reference orbit.

In the Great Circle geometry, the quadrupole is oriented so that $\hat{q}=\hat{k}$ for each element. This placement ensures that no linear coupling is introduced by quadrupole orientation.

In the Screw geometry, the quadrupole is placed with its symmetry axis along the direction of the reference particle orbit, i.e. at $\theta_{V}$ to the accelerator plane, and oriented so that the vertical quadrupole axis defines a plane intersecting the accelerator plane at the angle $\theta_{\mathrm{V}}$. The vector $\hat{\mathrm{q}}$ is therefore fixed by

$$
\hat{q} \cdot \hat{z}=\sin \theta_{v}
$$

and

$$
\hat{q} \cdot \hat{n}=0 \text {. }
$$


In this sequence, $\hat{q}$ is therefore not aligned with $\hat{k}$, the local vertical direction, so that linear coupling is introduced by this magnet placement. The strength of this coupling is measured by the angle $Y$, found from

$$
\hat{q} \cdot \hat{k}=\cos \gamma
$$

and can be estimated using the algorithm of the previous section. Quadrupoles in the Straight Section

Region IV is a region containing quadrupoles and no bending elements. The reference orbit direction is taken to be in the accelerator plane, that is, its component along $\hat{z}$ is zero. In either geometry, the Screw or Great Circle, the local coordinate frame is not upright, meaning $\hat{k} \neq \hat{z}$. Thus, if $\hat{q}$ is placed along $\hat{z}$, i.e. if the quadrupole is upright, then

$$
\hat{q} \cdot \hat{k}=\cos Y, \gamma \neq 0,
$$

and coupling is introduced.

We can, of course, simply place $\hat{q}$ along $\hat{k}$ and eliminate this contribution to linear coupling. However, another method to eliminate this coupling is to insert magnetic dipole fields along the reference orbit direction at the input and output of the straight section. This longitudinal or solenoidal field rotates the local reference frame around the reference orbit direction. If we rotate $\hat{k}$ at the input by $-\gamma$, making $\hat{k}$ and $\hat{z}$ coincident, then placing $\hat{q}$ along $\hat{z}$ introduces no coupling. At the end of the straight section, introduce another magnet, rotating $\hat{k}$ by $+\gamma$ and returning the local frame to its input orientation. This method of removing linear coupling along the slopes in the Screw geometry does not seem practical. For a longitudinal magnet of length $l$, the 
strength, $B_{L}$, required to obtain a coordinate frame rotation of angle $\gamma$ is given by

$$
\frac{B_{L} l}{B \rho}=\gamma .
$$

IV. Darticle Motion Around the Reference Orbit

Once a reference orbit is established, orbit stabilicy can be analyzed in terms of oscillations about this orbit. He refer to vertical oscillations for oscillations in the direction of the local vertical direction $\hat{k}$ and horizontal oscillations for oscillations in the direction of the local vector, $\hat{i}$. We use the coordinates $z, x$ and $s$ to denote distances along the axes $\hat{k}, \hat{i}$ and $\hat{n}$, respectively. Particles whose initial conditions deviate from those of the reference orbit will excute betatron motion about the reference orbit. Particles whose initial momenta along the reference particle direction are different from that of the reference orbit particle will exhibit chromatic properties and their betatron motion must be analyzed in terms of a displaced reference orbit determined by the momentum error.

\section{Vertical Dispersion}

The bending outside of the original accelerator plane introduces dispersion in both transverse directions. The dispersion is computed by introducing a bending field into the oscillation equations equal to the residual bending for an off-momentum particle. The vertical dispersion is the dispersion for the direction along the local $\hat{k}$ vector and this corresponds, outside the perturbed region, to the dispersion vertical to the accelerator plane.

To estimate the effect, we can, in the first instance, neglect the curvature in the plane of the bending relative to the magnet symetry axis, i.e. neglect the sagitta. For high energy beams, with bending per magnet small, this 
approximation is generally good. If we do this, the directions of the reference frame axes, $(\hat{k}, \hat{i}, \hat{n})$, do not change through the magnet. Of course, it is not necessary to impose this approximation; however, then we must integrate the fields in the directions $\hat{\mathbf{k}}$ and $\hat{\mathbf{i}}$ along the curved orbit in the magnet. Note that at different points along the trajectory through a dipole magnet, a field component along the longitudinal reference direction is introduced. But with the magnet placements we are employing, that is, where the direction of motion is parallel to the symmetry axis at the magnet center, then this longitudinal component averages to zero along the full magnet length:

Effect on Betatron Motion of Magnet Orientation with Respect to the Local

Vertical

if the $\hat{b}$ vector of a dipole or the $\hat{q}$ vector of a quadrupole is not coincident with the local $\hat{k}$, then a coupling of vertical and horizontal motion results. Thus the introduction of angles $\alpha$ and $\gamma$ not zero means coupling. In a quadrupole, $\hat{k}$ is unchanged through the magnet, and the field coupling terms are obtained as functions of a rotation angle $\gamma$ about $\hat{n}$. In a dipole magnet, the field decomposition along $(\hat{k}, \hat{i}, \hat{n})$ varies along the length of the magnet. To analyze the problem, we assume that the field components can be averaged along the reference path in the magnet independent of the changing values of the coordinates $(z, x, s)$ in the local frame. This approximation is good provided the betatron phase advance along the magnet is small, generally the case for high energy accelerators. Also, we can use the approximation, previously discussed for the dispersion analysis, of neglecting the reference orbit curvature in the magnet to further simplify the analysis. Again, if $\hat{b}$ and $\hat{\mathbf{k}}$ are not coincident, coupling of of the higher order field components 
of the dipoles results, with the magnitude of the coupling terms a function of the angle $\alpha$, with the coupling going to zero as $\alpha$ goes to zero.

V. Summary

We have provided a framework for the development of a beam model in the case of space orbits not confined to a plane. In particular, we have introduced an appropriate moving reference system for the analysis of beam stability. We have, as examples, considered two overpass geometries, the Great Circle and the Screw. The Great Circle sequence is specifically defined to preclude horizontal-vertical coupling arising from both quadrupole fields and higher order bending fields. The Screw sequence places a premium on accuracy of location of the magnets in the perturbed part of the lattice relative to the original acce1erator plane. Doing this, however, removes the fleedom of eliminating the coupling by magnet placement, the latter characterizing the Great Circle geometry. We have shown that the coupling strength introduced by each element in the screw sequence is second order in small angles; and that an estimate of the coupling can be computed using the algorithm for $\alpha_{\mathrm{J}}$. Since a longitudinal field rotates the axis of the local coordinate frame around the orbit direction, a (+B, -B) arrangement before and after a quadrupole sequence can allow independent quadrupole rotational placement. Such an arrangement could allow upright quadrupole placement in the straight section without introducing coupling, but is probably not a practical method for individual qudrupoles on the slopes. 